\title{
Correction to: Analysis of generalised alternating local discontinuous Galerkin method on layer-adapted mesh for singularly perturbed problems
}

\author{
Yao Cheng ${ }^{1}$ (1) $\cdot$ Yanjie $\mathrm{Mei}^{2}$
}

Published online: 7 February 2022

๑) The Author(s) under exclusive licence to Istituto di Informatica e Telematica (IIT) 2022

\section{Correction to: Calcolo (2021) 58:1-36 \\ https://doi.org/10.1007/s10092-021-00445-2}

The copyright holder for the article was incorrectly given as 'The Author(s), under exclusive licence to The Author(s) under exclusive licence to Istituto di Informatica e Telematica (IIT)' but should have been 'The Author(s) under exclusive licence to Istituto di Informatica e Telematica (IIT)'.

The original article has been corrected.

Publisher's Note Springer Nature remains neutral with regard to jurisdictional claims in published maps and institutional affiliations.

The original article can be found online at https://doi.org/10.1007/s10092-021-00445-2.

Yao Cheng

ycheng@usts.edu.cn

1 School of Mathematical Sciences, Suzhou University of Science and Technology, Jiangsu 215009, China

2 International Education School, Suzhou University of Science and Technology, Jiangsu 215009, China 